\title{
La télénivométrie et la prévision des apports de remplissage des réservoirs EDF
}

\author{
P. Tourasse
}

Electricité de France, Division Technique Générale, 37, rue Diderot, B.P. 41, 38040 Grenoble, France.

Les apports de fusion des stocks de neige de moyenne et haute altitude représentent une part importante des apports totaux annuels qui sont stockés ou qui transitent dans les réservoirs EDF - au total, plus de 7 milliards de $\mathrm{m}^{3}$ de capacité principalement dans les Alpes, les Pyrénées et le Massif-Central. Soumis à une très forte variabilité interannuelle, ces apports peuvent être prévus plusieurs mois à l'avance grâce à l'observation du manteau neigeux et à la mesure des précipitations d'hiver et de printemps.

C'est ainsi que, depuis de longues années, EDF optimise la gestion des grands lacs nationaux en calculant, dès le début de l'hiver et durant tout le printemps, des prévisions actualisées d'apports de fusion. Les mesures d'enneigement sont effectuées soit manuellement - chaque semaine ou chaque début de mois pour les sites les plus difficiles d'accès - soit automatiquement grâce à un réseau cohérent de télénivomètres qui mesurent en continu non seulement l'épaisseur mais surtout la densité et l'équivalent en eau du manteau neigeux.

Le réseau EDF de télénivomètres fonctionne de façon totalement opérationnelle depuis maintenant près de 20 ans. Il a permis, tout au long de ces années, d'améliorer considérablement la connaissance de la climatologie des zones de montagne.

Grâce à une fiabilité remarquable, ce réseau est un complément indispensable aux mesures manuelles qui sont souvent difficiles à réaliser en conditions extrêmes. Il permet de suivre au quotidien l'évolution des stocks de haute altitude et, à des pas de temps plus rapprochés — de une heure à quelques heures - , l'évolution plus rapide des stocks fluctuants de moyenne et basse altitude.

Associées à d'autres mesures hydrologiques - de précipitations, de température et de débits - et utilisées comme entrée dans des modèles de prévisions simples (enneigement-écoulement) ou combinés (précipitationstempérature-enneigement-écoulement), les données d'enneigement sont une aide très précieuse pour la gestion optimisée des moyens de production d'Electricité de France.

Même si les techniques de mesure sont amenées à évoluer - les normes réglementaires liées à l'utilisation de radioéléments sont de plus en plus strictes - le développement de la télénivométrie a constitué une base d'innovation avancée au cours des 20 dernières années. L'appareil, en lui-même, n'a pas vraiment d'équivalent à travers le monde et si le réseau français - avec une quarantaine de sites représentatifs équipés - peut être considéré comme à peu près équipé, de nouveaux réseaux, malgré l'importance des investissements à réaliser, sont en cours de constitution en Suisse et en Espagne.

Grâce à ces données - on dispose ainsi de chroniques historiques plus longues et plus détaillées - des progrès sensibles ont été réalisés, au fil des années, en matière de modélisation. Mais c'est surtout parce que l'automatisation de la collecte des données permet désormais de réactualiser les prévisions à chaque variation importante du stock que la télénivométrie constitue un progrès important pour la prévision opérationnelle des apports de fusion à long terme.

\section{LA TÉLÉNIVOMÉTRIE : UN COMPLÉ- MENT INDISPENSABLE POUR LE SUIVI DU MANTEAU NEIGEUX}

\subsection{Les mesures manuelles}

La mise sur pied du réseau de mesure d'enneigement d'EDF date du début des années cinquante à l'aube du développement du potentiel hydroélectrique français. Le réseau consistait en des mesures manuelles d'épaisseur et de densité en des emplacements témoins rigoureusement sélectionnés et repérés par des perches métalliques scellées dans le sol. Les mesures étaient pratiquées chaque semaine près des zones habitées, chaque début de mois aux altitudes plus élevées, voire seulement au début du mois d'avril pour les sites les plus difficiles d'accès.

A sa plus forte extension, au début des années soixante dix, ce réseau a compté jusqu'à 600 points de mesure [1]. Même si de nombreuses séries ont depuis lors été arrêtées, on avait jeté là les bases de la nivoclimatologie de montagne et commencé à constituer au fil de ces 20 premières années un véritable patrimoine de séries de mesures irremplaçables (voir tabl. 1).

Il s'agissait là du premier véritable réseau de mesure de l'enneigement en France [2], car les quelques points existants avant guerre n'avaient pas fait l'objet de suivi régulier ni de procédures de mesure vraiment unifiées. Les séries les plus anciennes remontent au début des années 50 - on pense notamment à la série de Serre-Chevalier sur la haute-Durance qui a démarré en 1948 et qui constitue un excellent témoin des apports de fusion dans Serre-Ponçon - et permettent aujourd'hui de disposer de chroniques critiquées de près de 45 ans.

Dans les années soixante dix, devant les difficultés de recrutement d'observateurs capables de réaliser en sécurité 
Tableau 1. - Données d'enneigement disponibles sur les sites abandonnés du bassin de la Durance.

\begin{tabular}{|c|c|c|c|c|c|}
\hline Perches & $\begin{array}{c}\text { Course ou } \\
\text { massif }\end{array}$ & $\begin{array}{l}\text { Alt. } \\
\text { (m) }\end{array}$ & $\begin{array}{c}\text { Fréquence } \\
\text { mesures }\end{array}$ & $\begin{array}{l}\text { Date } \\
\text { debut }\end{array}$ & $\begin{array}{c}\text { Date } \\
\text { fin }\end{array}$ \\
\hline $\begin{array}{l}\text { PALUEL } \\
\text { LA LOUYERE } \\
\text { FARAVEL } \\
\text { LAC DU SERPENT } \\
\text { LARAMON } \\
\text { CABANE DES BANS } \\
\text { BLOCKAUS } \\
\text { GRGES DU GALIBIER } \\
\text { MANDETTE } \\
\text { SESTRIERE } \\
\text { ROCHE NOIRE } \\
\text { L'ALP DE LA CIME } \\
\text { PRA DU LAUS } \\
\text { TABUC } \\
\text { LA SALLE } \\
\text { CLOT DE L'ARPELIN } \\
\text { COL PERDU } \\
\text { CASSE DES OULES } \\
\text { FONTANIL } \\
\text { LES CLAUX } \\
\text { LELAUS } \\
\text { ENTRE LES AIGUES } \\
\text { PRA-FAUCHIER } \\
\text { SERRE DES EYNARDS } \\
\text { GIRARDES } \\
\text { PETIT LAC } \\
\text { LELAUS } \\
\text { CABANE DE L'OURS } \\
\text { PONT DE PIERRE } \\
\text { NEVACHE } \\
\text { SAUME LONGUE }\end{array}$ & $\begin{array}{c}\text { Galibier } \\
\text { Cerveyrette } \\
\text { Izoard } \\
\vdots \\
" \\
"\end{array}$ & $\begin{array}{l}2240 \\
2250 \\
2170 \\
2435 \\
2340 \\
1780 \\
2550 \\
2355 \\
2290 \\
1930 \\
2010 \\
2120 \\
2200 \\
2180 \\
2370 \\
2150 \\
2430 \\
2310 \\
2120 \\
1250 \\
1760 \\
1570 \\
1940 \\
2050 \\
2420 \\
1840 \\
2580 \\
2460 \\
2155 \\
1590 \\
2240\end{array}$ & 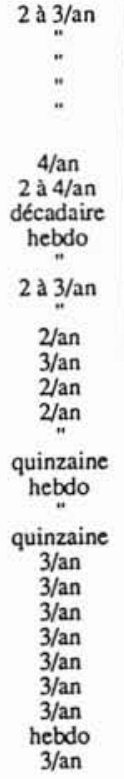 & $\begin{array}{l}57 \\
57 \\
57 \\
56 \\
56 \\
53 \\
56 \\
56 \\
53 \\
51 \\
59 \\
58 \\
58 \\
60 \\
52 \\
56 \\
56 \\
56 \\
56 \\
51 \\
60 \\
52 \\
60 \\
60 \\
60 \\
61 \\
56 \\
56 \\
56 \\
60 \\
56\end{array}$ & $\begin{array}{l}75 \\
75 \\
75 \\
80 \\
80 \\
60 \\
84 \\
65 \\
84 \\
58 \\
84 \\
71 \\
71 \\
75 \\
78 \\
74 \\
72 \\
72 \\
81 \\
71 \\
81 \\
70 \\
71 \\
71 \\
71 \\
71 \\
63 \\
71 \\
71 \\
79 \\
78\end{array}$ \\
\hline
\end{tabular}

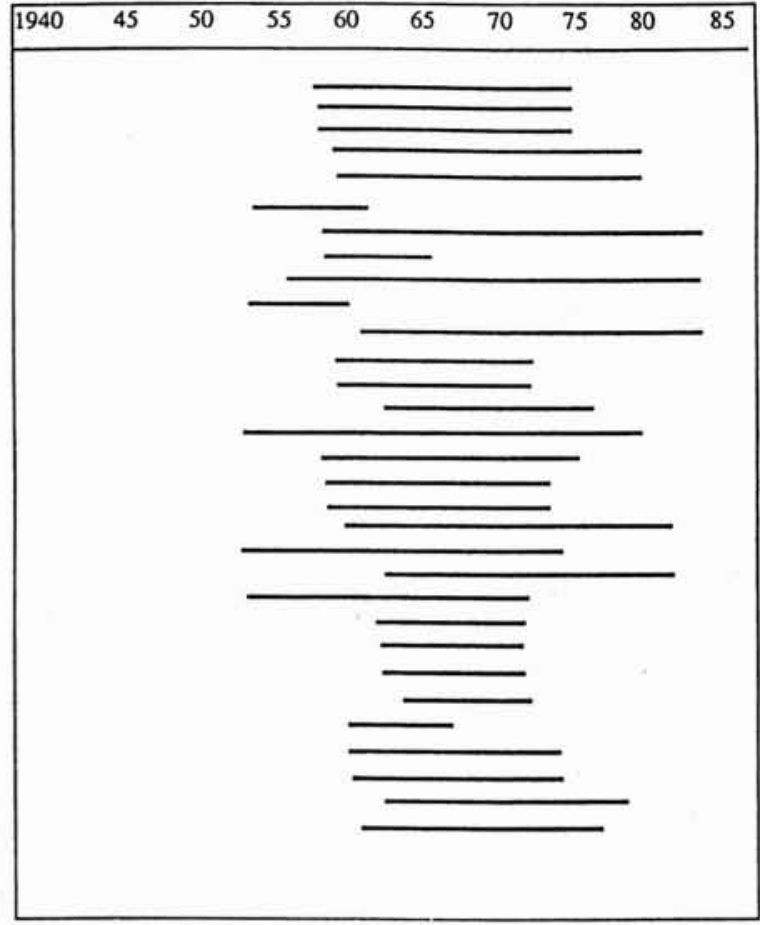

des mesures de bonne qualité mais surtout aussi pour des raisons économiques, EDF a entrepris de rationnaliser cet important dispositif d'observation. Une analyse approfondie des mesures historiques a été entreprise sur l'ensemble des massifs des Alpes, des Pyrénées et du Massif-Central. Elle a été complétée par l'étude systématique des corrélations entre l'enneigement et les apports de fusion dans les rivières. Ces études ont permis de sélectionner, parmi tous les points existants, ceux qui étaient les plus représentatifs et de ramener ainsi le réseau à un nombre optimal de 240 emplacements tous situés entre 800 et 2700 mètres d'altitude.

Parallèlement et dès 1964, EDF avait commencé à étudier un dispositif automatique de mesure de la hauteur de neige et de son équivalent en eau, ce qui amena le développement d'une nouvelle génération d'appareils : les télénivomètres.

\subsection{Les mesures automatiques : les télénivomètres}

Le principe de mesure repose sur l'absorption d'un rayonnement à travers la couche de neige. Le rayonnement est fourni par une source radioactive de faible activité et son atténuation est mesurée par un compteur Geiger-Müller selon une fonction exponentielle décroissante de l'équivalent en eau de la couche de neige indépendamment de ses caractéristiques physiques et de son état cristallin. On mesure, en fait, le temps $T$ nécessaire pour compter un nombre fini de décharges du compteur et l'on déduit la valeur en eau de la couche de neige par une relation de la forme :

$$
V e=C \log \left(T / T_{0}\right)
$$

Il existe, aujourd'hui, 2 types d'appareils : le télénivomètre à faisceau vertical pour une mesure directe de l'équivalent en eau d'un manteau de faible épaisseur, le télénivomètre à faisceau horizontal pour une mesure couche par couche des manteaux neigeux de haute altitude.

\section{Le télénivomètre à faisceau vertical}

L'appareil est simple dans sa conception - il ne comporte, en particulier, aucune partie mobile - et permet de mesurer avec une bonne précision des couches de neige qui vont de quelques centimètres à quelque 2 mètres.

Il fournit directement l'équivalent en eau de la couche de neige avec une mesure associée de la hauteur de neige par ultrasons. Cet appareil qui est utilisé en moyenne montagne entre 900 et 1500 mètres n'influence pas le manteau neigeux.

Quotidiennement, les valeurs en eau et hauteurs de neige sont mesurées toutes les quatre heures ; un suivi horaire de la température de l'air autorise une surveillance fine de l'évolution de l'isotherme $0{ }^{\circ} \mathrm{C}$ à des altitudes où les phénomènes de fusion peuvent, dans certains cas, conduire à un lessivage rapide du manteau.

\section{Le télénivomètre à faisceau horizontal mobile}

Il mesure la densité du manteau neigeux par tranches de 10 centimètres et fournit, par sommation, la valeur en eau et l'épaisseur totale du manteau.

Il ne demande qu'une source radioactive de très faible activité $(10 \mathrm{mCi})$ qui n'émerge de la neige que quelques secondes par jour et d'une dizaine de centimètres seulement. L'ensemble source-détecteur se déplace verticalement à travers le manteau neigeux dont l'épaisseur peut aller jusqu'à 6 mètres. 
Il fournit une mesure par jour - soit l'équivalent de près de 200 sondages par hiver - et donne à chaque mesure une description fine des densités des différentes couches du manteau ; ce qui peut être très utile pour certaines applications et s'avère précieux pour la validation des données.

Chaque appareil est autonome en énergie grâce à un dispositif d'alimentation par batterie et panneau solaire. Malgré sa technologie sophistiquée le télénivomètre a été conçu pour supporter des conditions extrêmes et peut fonctionner sans visite pendant toute la saison d'hiver. Grâce à une normalisation poussée des procédures de fabrication, la fiabilité atteinte est très bonne - moins d'une à deux pannes par an en moyenne sur tout le parc EDF - et il n'est pas rare qu'un appareil fonctionne plusieurs années de suite sans incident et sans nécessiter d'autre intervention que de routine.

\subsection{Un ensemble cohérent de 40 sites automatisés}

Si la collecte automatisée des données hydrologiques a bénéficié de progrès spectaculaires au cours des 10 dernières années, la télécollecte des données d'enneigement a joué un rôle précurseur dans ce domaine et fait longtemps figure de référence [3]. Les données furent d'abord récupérées par simple voie hertzienne mais la transmission radio devait rapidement céder la place au satellite ARGOS qui outre sa remarquable fiabilité - les pertes d'informations par défaut de transmission sur le réseau ARGOS sont quasi inexistantes - permet de concentrer plus facilement, en un point central, les données des sites les plus éloignés. Pour les sites de moyenne altitude qui demandent à être interrogés plusieurs fois par jour, le téléphone reste le moyen le plus souple et le moins onéreux. Mais il souffre encore de faiblesses endémiques dans certaines zones rurales mal desservies [4].

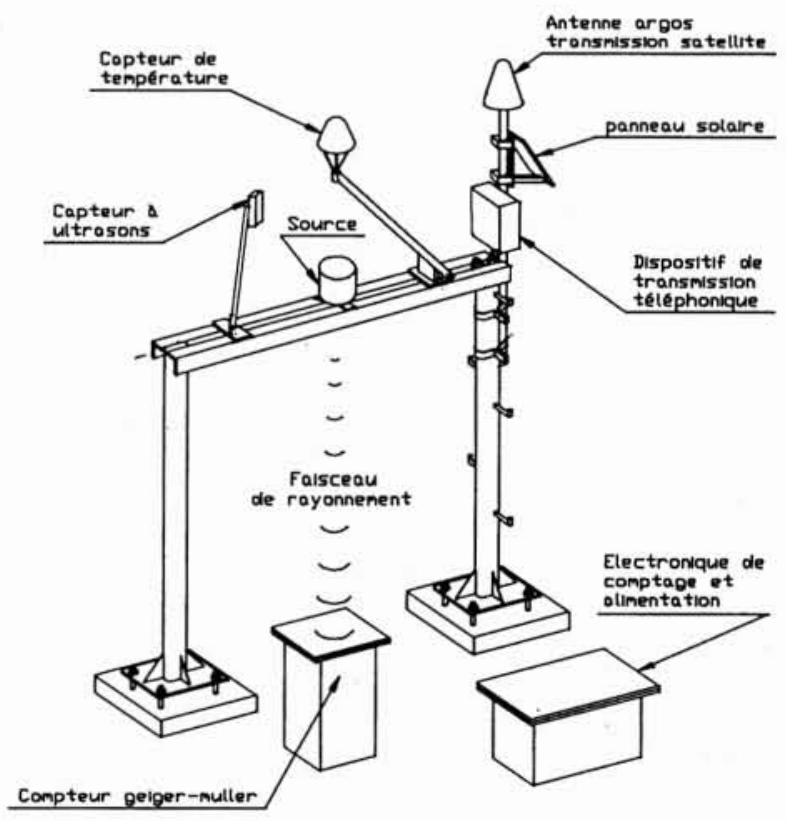

SCHEMA DU TELENIVOMETRE VERTICAL
A ce jour (fig. 2) près de 40 sites sont équipés de télénivomètres: une trentaine en haute altitude et une dizaine sur les bassins de moyenne altitude du Jura, des Préalpes et du Massif-Central. Les sites ont été choisis en fonction de leur représentativité [5], l'ensemble formant un tout cohérent qui complète les mesures manuelles et permet de disposer en permanence d'une photographie actualisée de l'état du manteau neigeux pour les bassins dont les rivières participent, au printemps, au remplissage des réservoirs EDF.

\subsection{Enseignements et perspectives}

Après plusieurs phases d'évolution sensible pendant les premières années d'exploitation, le parc EDF des télénivomètres s'est stabilisé au début des années 80 ; tant sur le plan technologique que par le nombre de stations en exploitation. On dispose ainsi maintenant d'une bonne quinzaine d'années d'expérience de ce type d'appareil avec comme principaux enseignements :

- sa remarquable fiabilité : tel qu'il est conçu, l'appareil supporte bien les conditions extrêmes auxquelles il est soumis pendant toute la période hivernale. Il est peu sensible également - parce que non relié au réseau EDF ni au réseau PTT - aux perturbations électromagnétiques qui sont un souci permanent pour l'exploitation des systèmes automatiques de collecte des données hydrologiques ;

- le caractère irremplaçable d'informations qu'il apporte sur la valeur en eau du manteau neigeux qui seule est bien corrélée avec les apports de fusion que l'on cherche à prévoir surtout le fait que les données sont disponibles très rapidement - une fois par jour pour les stocks de haute altitude, toutes les quatre heures pour le suivi des manteaux neigeux de moyenne et basse altitude - ce qui permet de réactualiser au plus vite les prévisions lorsque le stock de

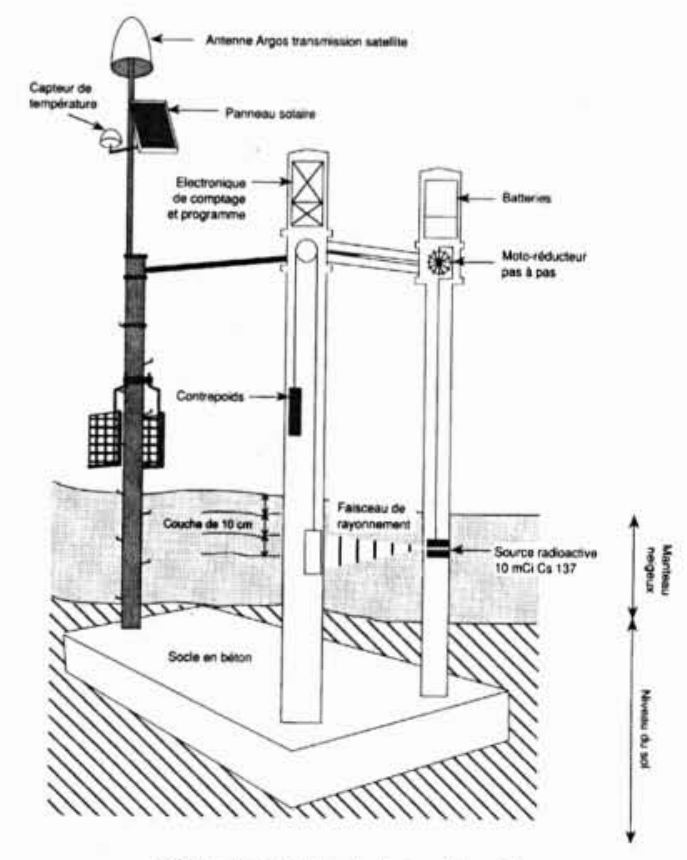

Schéma du téléniviomètre horizontal mobile

1. Deux appareils de conception différente pour suivre l'enneigement de moyenne et de haute altitude. 


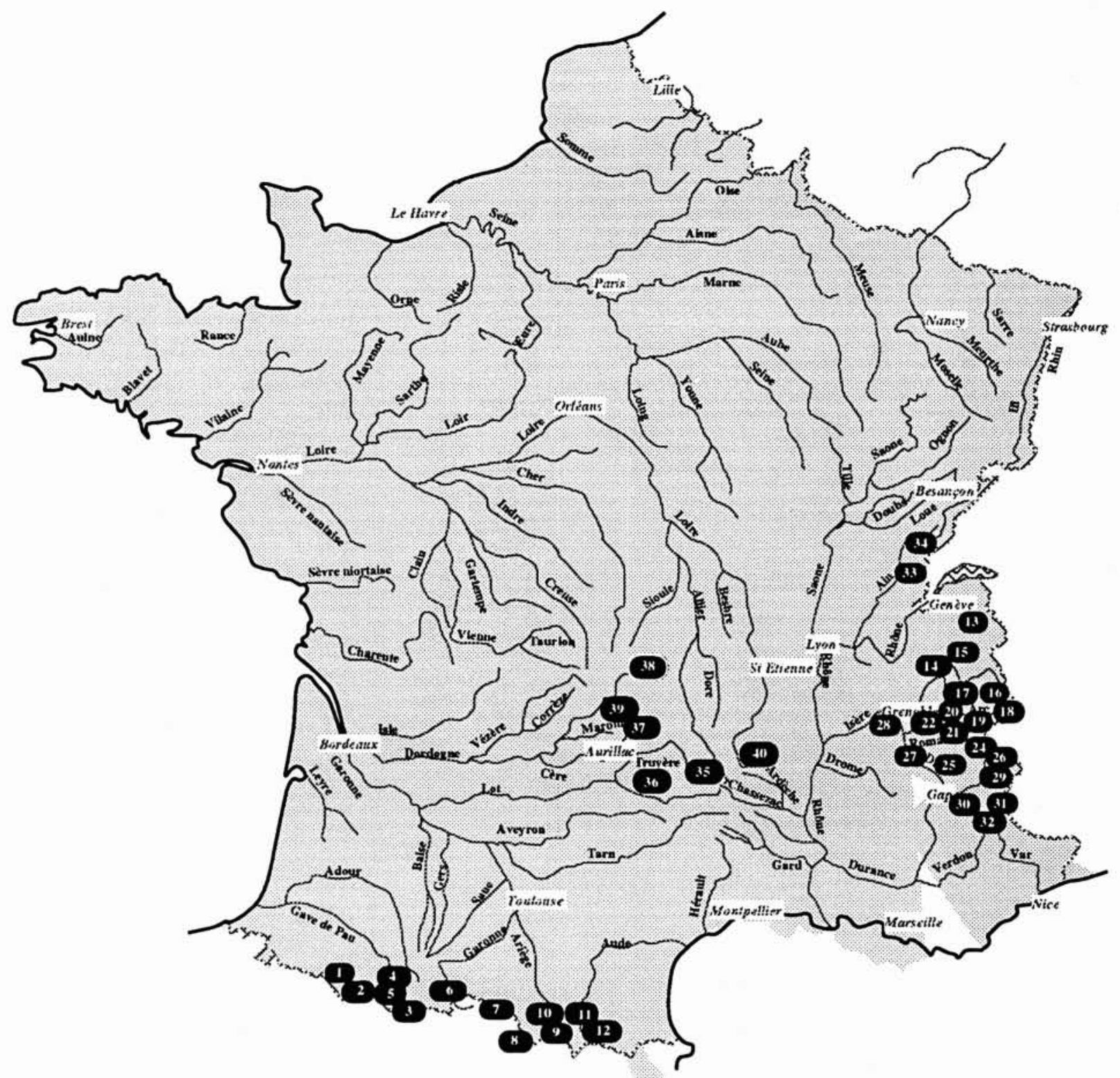

2. Le réseau des télénivomètres EDF : un ensemble cohérent de 40 appareils automatiques.

neige subit une variation importante. Il ne faut pas oublier, en effet, que les sondages manuels sont difficiles à effectuer en haute altitude ; les courses sont longues et les conditions d'approche sont souvent délicates, voire même dangereuses ;

- la difficulté qui persiste à conserver une bonne représentativité de la mesure en fin de printemps quand la mesure ponctuelle pert de sa signification sous l'influence de l'exposition et des particularités du terrain. On observe aussi la formation de cônes de fusion autour des poteaux de l'appareil et la perte d'information qui en découle par sous-estimation de la valeur en eau du profil — devient de plus en plus significative au fur et à mesure que l'on avance dans la fusion.
Dans ce contexte, l'évaluation des surfaces qui restent enneigées lorsque la fusion est déjà bien amorcée, est d'un grand intérêt pour la prévision des apports résiduels de fin de printemps et de début d'été.

Le simple survol du bassin versant par des moyens héliportés ou par avion peut être suffisant si l'on s'intéresse à un bassin de faible dimension et si l'on peut se contenter d'une simple information qualitative. Il s'agit alors simplement d'évaluer s'il reste un stock résiduel significatif alors que les mesures ponctuelles - parce que situées trop bas en altitude ou en des points trop exposés - ne donnent plus aucune information.

Le recours à l'imagerie satellitaire de type LANDSAT ou SPOT est une voie qui mérite d'être explorée. On bute 
toutefois sur de réelles difficultés de mise en œuvre opérationnelle et sur des coûts qui, à ce jour, ne permettent pas d'envisager une utilisation régulière et à grande échelle de ce type d'information.

Dans le cas du satellite LANDSAT, la définition est excellente. On peut disposer d'une image environ tous les quinze jours mais celles-ci ne sont pas programmables et souvent altérées par les formations nuageuses qui cachent complètement le terrain ou s'accrochent au relief.

Dans le cas du satellite SPOT, il est possible d'obtenir des images plus fréquentes grâce à la programmation des caméras. Mais le coût de l'image programmée est très important.

D'une manière générale - une campagne d'étude portant sur la période 1986-1991 a été réalisée sur la hauteDurance (fig. 3) —, l'expérience montre que l'on ne peut vraiment traiter qu'une à deux images par saison et que celles-ci ne correspondent pas toujours aux périodes les plus intéressantes. Les délais de mise à disposition des

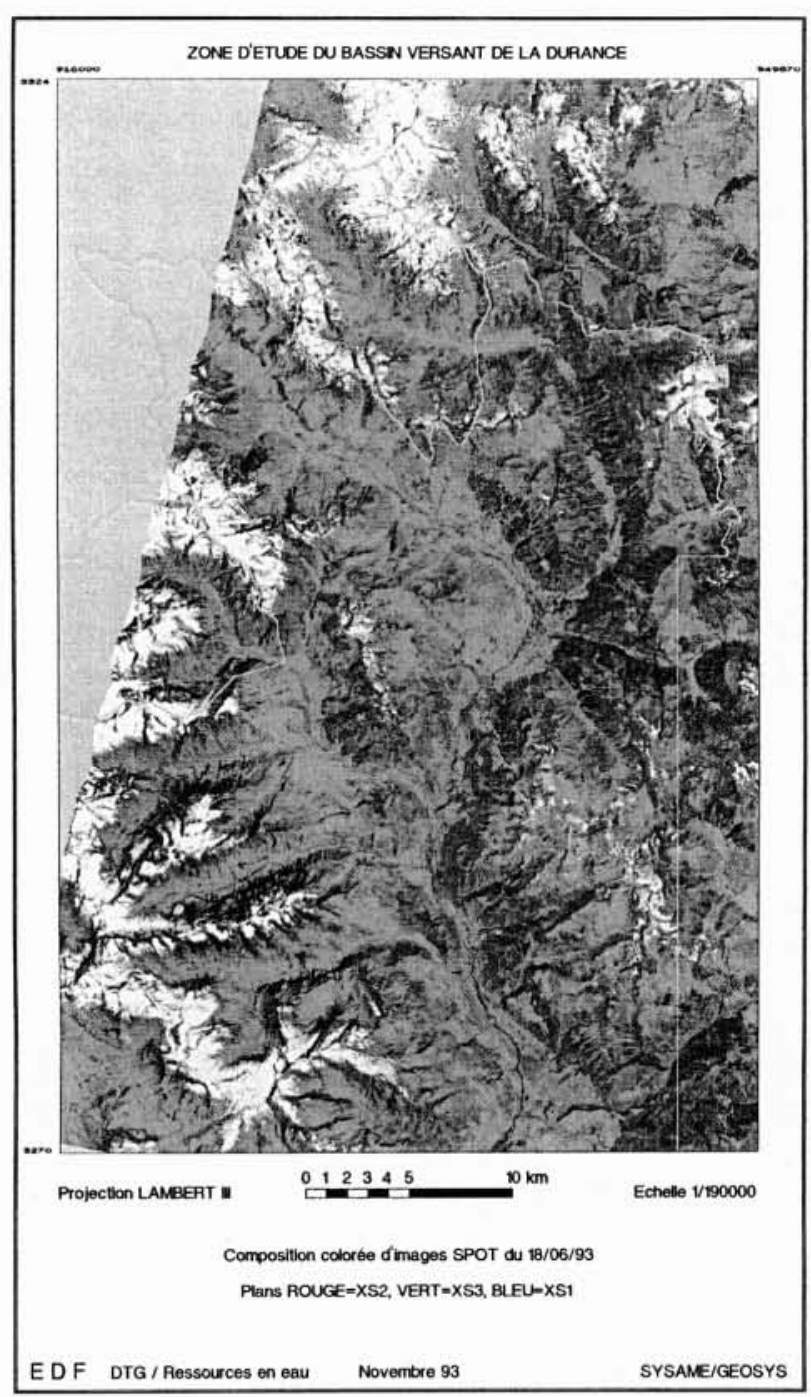

3. Imagerie satellitaire de l'enneigement de la haute Durance. images sont également importants ce qui ne va pas, non plus, dans le sens d'une grande opérationnalité.

Malgré cela, il paraît indispensable de poursuivre les efforts de recherche dans ce domaine. Les moyens de traitement vont encore progresser et l'utilisation de l'imagerie satellitaire devrait se développer dans l'avenir. Celle-ci restera toutefois limitée à la seule période de fusion car la corrélation entre la surface enneigée et l'apport de fonte n'est évidemment pas pertinente en début d'hiver quand tout le bassin est enneigé ni, d'une manière générale, pendant toute la période d'accumulation.

\section{II — UTILISATION DES DONNÉES D'ENNEI- GEMENT DANS LES PRÉVISIONS À LONG TERME}

Pour tous les bassins dont le régime a une composante nivale importante avec une forte abondance et une grande variabilité interrannuelle des apports de printemps, il est possible de prévoir, dès le début de l'hiver, les apports cumulés des mois d'avril à juillet ou ceux d'avril à août pour les bassins les plus enneigés. On s'appuie sur les fortes corrélations qui existent entre les précipitations cumulées d'hiver et les apports de printemps, ou entre l'enneigement de début d'hiver et les apports de fusion.

Les modèles utilisés de façon opérationnelle sont choisis pour leur robustesse. Ils ne font intervenir qu'un nombre limité de préviseurs - des précipitations, de l'enneigement de haute altitude, éventuellement des débits ou des températures - en les agrégeant à des pas mensuels ou plurimensuels. Les prévisions sont émises chaque début de

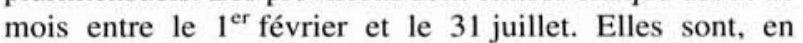
général en bonne adéquation avec les valeurs réellement observées, malgré un aléa résiduel qui dépend, en grande partie, des précipitations de printemps qui ne sont pas connues au moment où l'on effectue la prévision et qui peuvent, certaines années, s'écarter notoirement de la normale.

\subsection{La Durance à Serre-Ponçon}

Dans le cas de la Durance à Serre-Ponçon, les mesures manuelles d'enneigement sont effectuées chaque hiver en une vingtaine de points représentatifs (fig. 4). Cinq télénivomètres respectivement situés à Chardonnet sur la Guisane, Cézanne au pré de Madame Carle, Izoard, les Marrous sur le Haut-Guil et Passaur sur l'Ubaye complètent ce dispositif et permettent de connaître, chaque jour, par télémesure, l'évolution du manteau neigeux sur l'ensemble du bassin.

Les prévisions d'apports de remplissage du réservoir de Serre-Ponçon sont calculées et réactualisées toutes les semaines du $1^{\text {er }}$ février au $1^{\text {er }}$ juillet. Les données utilisées sont les mesures d'enneigement des trois sites les plus représentatifs (Cézanne, Serre-Chevalier et Izoard) et les mesures de précipitations d'hiver et de printemps d'une sélection de 8 stations. Mais l'estimation de l'état des réserves hydriques du sol constituent aussi un élément complémentaire important pour apprécier le rendement des écoulements de printemps et d'été.

Les prévisions émises sont, en général, en bonne adéquation avec les valeurs observées (fig. 5). 
BASSIN DE LA DURANCE A SERRE-PONCON (3600 km2)

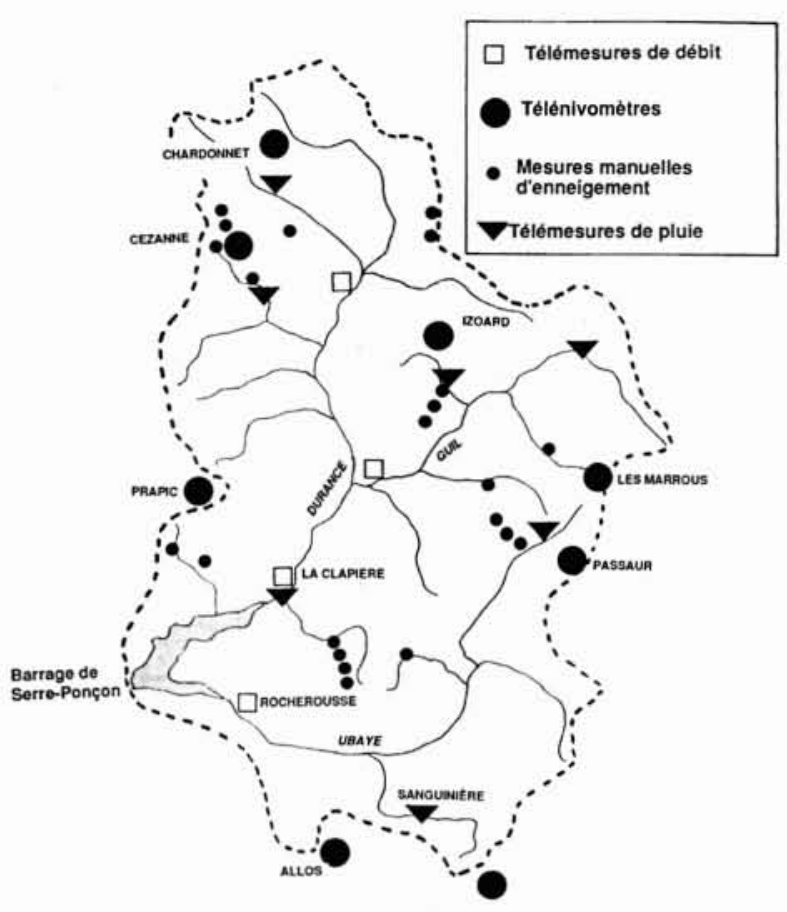

4. Mesures d'enneigement en amont de Serre-Ponçon.

Toutefois, certaines années - ce fut le cas durant l'été 90 - les apports entrant dans la retenue entre le $1^{\text {er }}$ avril et le 31 juillet peuvent être inférieurs à la prévision la plus pessimiste (prévision à une chance sur 10). C'est dire que le seul déficit des précipitations et de l'enneigement de l'hiver ne suffit pas à expliquer le déficit des apports du printemps-été. Dans ce cas, plusieurs facteurs peuvent jouer: la recharge des réserves souterraines - elles avaient été particulièrement affectées par le déficit pluviométrique de l'année 89 - peut en être un.

Il en est de même lorsque l'enneigement a une distribution spatiale particulière - par exemple avec un fort différentiel entre le bassin de l'Ubaye et celui de la branche Durance ; ou lorsqu'on a une importante hétérogénéité du manteau avec l'altitude - et que les modèles sont pris en défaut à certaines échéances.

Dans ce cas, la connaissance du terrain est essentielle. La description fine de l'enneigement sur les bassins et sous-bassins qui contribuent à la formation des écoulements principaux constitue une aide très précieuse pour le

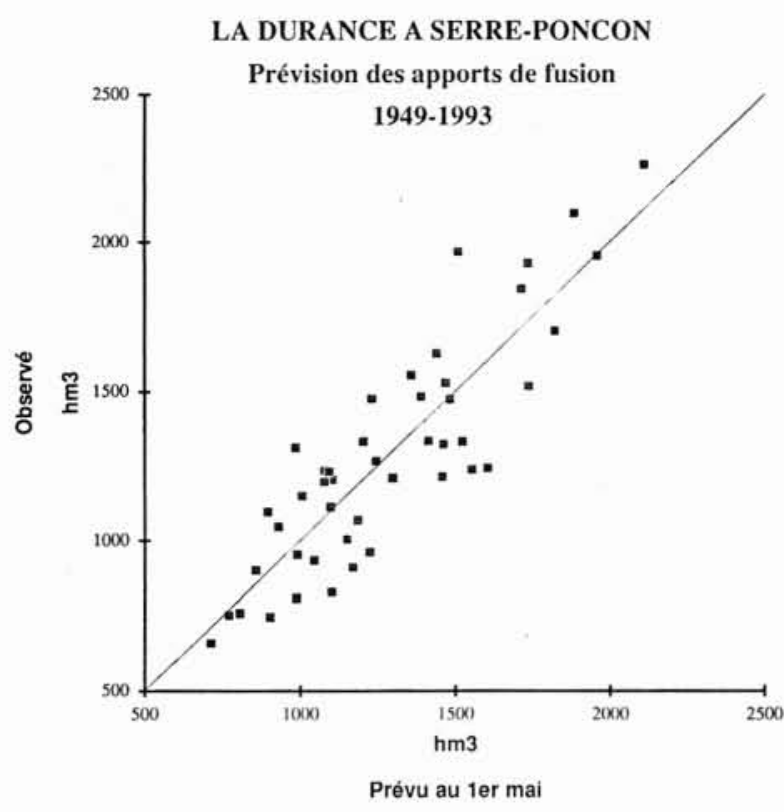

5. Prévisions au $1^{\text {er }}$ mai des apports entrant dans le réservoir de Serre-Ponçon entre le $1^{\text {er }}$ mai et le 31 juillet. Comparaison entre les apports observés (axe des ordonnnées) et les apports calculés par le modèle (axe des abscisses).

prévisionniste qui, en permanence, doit avoir en tête, d'une part la sensibilité des outils et des modèles qu'il utilise, d'autre part une photographie aussi fidèle que possible de la ressource à travers les index qui la représente.

\section{Références}

[1] Duband D. (1980). - 40 années de mesures d'enneigement à EDF, Journées SHF de la section de Glaciologie, Grenoble, février 1988.

[2] Duband D. et Lallement Ch. (1992). - 40 years of snow cover measurement in France : network evaluation and forecast interest, Western Snow Conference, Jackson Hole, Wyoming - USA, avril 1992.

[3] Guillot P. (1982). - Mesure de l'équivalent en eau de la couche de neige. Journées SHF de la section de Glaciologie, Paris, mars 1982.

[4] Duband D. et Tourasse P. (1992). - Progrès en cours et prévisibles en hydrologie et climatologie opérationnelles, $22^{e}$ Journées de l'hydraulique, Paris, septembre 1992.

[5] PINTE J. (1981). - Choix d'emplacements représentatifs de l'enneigement dans les Alpes du Nord, Note EDF-DTG, Grenoble, mars 1981. 